\title{
SOME INTEGRABILITY THEOREMS
}

\author{
by YUNG-MING CHEN
}

(Received 3 June, 1964; and in revised form 5 January, 1965)

1. P. Heywood [3] proved the following theorems:

THEOREM A. If $0 \leqq \gamma<2$, if $x^{\gamma-1} g(x) \in L(0, \pi)$, and if

$$
b_{n}=\frac{2}{\pi} \int_{0}^{\pi} g(x) \sin n x d x
$$

for $n=1,2,3, \ldots$, then the series $\sum_{1}^{\infty} n^{-\gamma} b_{n}$ is convergent.

THEOREM B. If $0 \leqq \gamma<1$, if $x^{\gamma-1} f(x) \in L(0, \pi)$, and if

$$
a_{n}=\frac{2}{\pi} \int_{0}^{\pi} f(x) \cos n x d x
$$

for $n=1,2,3, \ldots$, then the series $\sum_{1}^{\infty} n^{-v} a_{n}$ is convergent.

THEOREM C. Suppose that $g(x) \in L(0, \pi)$, that $b_{n}$ is defined by (1.1) for each $n$, that $0<\gamma \leqq 1$, and that the series $\sum_{1}^{\infty} n^{\gamma-1}\left|b_{n}\right|$ converges. Then the integral

$$
\int_{\rightarrow 0}^{\pi} x^{-y} g(x) d x
$$

exists as a Cauchy-Lebesgue integral.

THEOREM D. Suppose that $f(x) \in L(0, \pi)$, that $a_{n}$ is defined by (1.2) for $n=1,2, \ldots$, that $0<\gamma<1$, and that the series $\sum_{1}^{\infty} n^{\gamma-1}\left|a_{n}\right|$ converges. Then the integral

exists as a Cauchy-Lebesgue integral.

$$
\int_{\rightarrow 0}^{\pi} x^{-\gamma} f(x) d x
$$

When the index $\gamma$ satisfies $1<\gamma<2$, Siobhan O'Shea [6] has proved the following theorem:

Theorem E. Suppose that $1<\gamma<2$. Then the series

$$
\sum_{n=1}^{\infty} b_{n} \sin n x \quad\left(b_{n} \geqq 0\right)
$$

converges everywhere to a function $g(x)$ satisfying $x^{-\gamma} g(x) \in L(0, \pi)$, if and only if $\sum_{1}^{\infty} n^{\gamma-1} b_{n}<\infty$. 
The present note is concerned with generalizations of these theorems. We shall make use of a class of asymptotic functions which have previously been defined in [2]. By $\phi(x) \sim[a, b]$, $0 \leqq a \leqq b<\infty$ or $-\infty<a \leqq b \leqq 0$, we denote a non-negative function $\phi(x)$, not identically zero, such that $x^{-a} \phi(x)$ is non-decreasing and $x^{-b} \phi(x)$ is non-increasing, as $x$ increases in $(0, \infty)$. By $\phi(x) \sim\langle a, b\rangle$, we denote $\phi(x)$ such that there exists some positive constant $\varepsilon$ for which $\phi(x) \sim[a+\varepsilon, b-\varepsilon]$. We define $\phi(x) \sim[a, b\rangle$ and $\phi(x) \sim\langle a, b]$ in a similar way. We shall establish the following theorems:

TheOREM 1. If $x^{-1} \phi\left(x^{-1}\right) g(x) \in L(0, \pi)$, where $\phi(x) \sim[-1,0\rangle$ or $\phi(x) \sim\langle-2,-1\rangle$, and if $b_{n}$ is defined by (1.1) for $n=1,2,3, \ldots$, then $\sum_{1}^{\infty} \phi(n) b_{n}$ is convergent.

THEOREM 2. If $\phi(x) \sim\langle-1,0\rangle$ and $x^{-1} \phi\left(x^{-1}\right) f(x) \in L(0, \pi)$, and if $a_{n}$ is defined by (1.2) for every $n$, then $\sum_{1}^{\infty} \phi(n) a_{n}$ is convergent.

THEOREM 3. Suppose that $g(x) \in L(0, \pi)$, that $b_{n}$ is defined by $(1.1)$ for $n=1,2,3, \ldots$, and that $\sum_{1}^{\infty} n^{-1} \phi\left(n^{-1}\right)\left|b_{n}\right|<\infty$, where $\phi(x) \sim\langle-2,0\rangle$; then the integral

$$
\int_{\rightarrow 0}^{\pi} \phi(x) g(x) d x
$$

exists as a Cauchy-Lebesgue integral.

THEOREM 4. If $f(x) \in L(0, \pi)$, and if $a_{n}$ is defined by (1.2) for $n=1,2,3, \ldots$, such that $\sum_{1}^{\infty} n^{-1} \phi\left(n^{-1}\right)\left|a_{n}\right|<\infty$, where $\phi(x) \sim\langle-1,0\rangle$, then the integral

$$
\int_{\rightarrow 0}^{\pi} \phi(x) f(x) d x
$$

exists as a Cauchy-Lebesgue integral.

THEOREM 5. Let $\phi(x) \sim\langle-2,-1\rangle$, and let $b_{n} \geqq 0$ for every $n$. Then the trigonometric series $\sum_{1}^{\infty} b_{n} \sin n x$ converges everywhere to $g(x)$ such that $\phi(x) g(x) \in L(0, \pi)$, if and only if $\sum_{1}^{\infty} n^{-1} \phi\left(n^{-1}\right) b_{n}<\infty$.

2. It is natural to inquire whether the result in Theorem $A$ can be extended to integrability of the function $x^{-\gamma}\{g(x)\}^{p}$ for $p>1$ (cf. Math. Z. 66 (1956), 9-12). The answer is in the negative even when $p=2$. This may be justified by the example: $g(x)=(\pi-x)^{-t}$. Here we have $\pi b_{n} / 2 \simeq K(-1)^{n+1} n^{-\frac{7}{3}}$, so that if $\gamma=\frac{1}{3}, p=2$, then $x^{-\gamma}\{g(x)\}^{2} \in L(0, \pi)$, but $\sum_{1}^{\infty} n^{y} b_{n}^{p}=\sum_{1}^{\infty} n^{\frac{1}{3}} b_{n}^{2}=\infty$.

As a particular case in Theorem 1 , we may set $\phi(x)=x^{-\gamma} L(1 / x)$, where $0<\gamma<2$ and $L(t)$ is a slowly increasing function in the sense of Karamata $([4],[5]$; cf. also [7, p. 186]). 
Similar conditions may be applied to Theorems 2 to 5. By $a(x) \simeq b(x)$ and $a(x) \asymp b(x)$, as $x \rightarrow c$, we mean $a(x) / b(x) \rightarrow 1$ and $K_{1}<a(x) / b(x)<K_{2}$, respectively, as $x \rightarrow c$. Here and later the letter $K$ denotes a positive constant, not necessarily the same at each occurrence.

LemMa 1. Let

$$
g(x)=\sum_{1}^{\infty} \lambda_{n} \sin n x
$$

where $\lambda_{n}$ decreases steadily to zero. If $\phi(x) \sim[-1,0\rangle$ and if $\lambda_{n} \simeq \phi(n)$ as $n \rightarrow \infty$, then $g(x) \asymp x^{-1} \phi\left(x^{-1}\right)$, as $x \rightarrow+0$.

Proof. Since $\phi(x) \sim[-1,0\rangle$, by Lemma 1 in [2], $\phi(x)$ is absolutely continuous in $(\delta, \infty)$, where $\phi(x)$ decreases monotonically. From $\phi(x) \sim[-1,0\rangle$, we obtain $x \phi(x) \sim[0,1\rangle$, where $x \phi(x)$ is non-decreasing in $(0, \infty)$. It follows that

where

$$
g(x)=\sum_{1}^{\infty} \lambda_{n} \sin n x=\sum_{1 \leqq n \leqq[1 / x]}+\sum_{n>[1 / x]}=S_{1}+S_{2},
$$

$$
\left|S_{1}\right| \leqq K x\left|\sum_{1}^{[1 / x]} n \lambda_{n}\right| \leqq K x \int_{1}^{1 / x} t \phi(t) d t \leqq K x\left(\frac{1}{x}\right)\left\{\frac{1}{x} \phi\left(\frac{1}{x}\right)\right\}=\frac{K}{x} \phi\left(\frac{1}{x}\right) .
$$

By Abel's transformation, it is easy to verify that

$$
\left|S_{2}\right|=\left|\sum_{n>1 / x} \lambda_{n} \sin n x\right| \leqq \frac{K}{x} \phi\left(\frac{1}{x}\right)
$$

It remains to show that $g(x)>K x^{-1} \phi\left(x^{-1}\right)$, as $x \rightarrow+0$. To see this, write

$$
\left.\begin{array}{rl}
g(x) & =\sum_{1}^{\infty} \Delta \lambda_{n} \frac{\sin ^{2} \frac{1}{2}\left(n+\frac{1}{2}\right) x}{\sin \frac{1}{2} x}-\frac{\lambda_{1}}{2} \tan \frac{x}{4} \\
& =\sum_{1}^{\infty} \Delta \lambda_{n} \frac{\sin ^{2} \frac{1}{2}\left(n+\frac{1}{2}\right) x}{\sin \frac{1}{2} x}+o\left\{\frac{1}{x} \phi\left(\frac{1}{x}\right)\right\}=S_{3}+S_{4},
\end{array}\right\}
$$

say. If $\phi(x) \sim\langle-m, 0\rangle$, then $x^{\varepsilon} \phi(x)$ decreases and $x^{m} \phi(x)$ increases for some $\varepsilon>0$ in $(0, \infty)$. This implies that $n^{\varepsilon} \phi(n)>(2 n)^{\varepsilon} \phi(2 n)$ and $n^{m} \phi(n)<(2 n)^{m} \phi(2 n)$. It follows that $\phi(n)-\phi(2 n)>\left(2^{i}-1\right) \phi(2 n)>2^{-m}\left(2^{e}-1\right) \phi(n)=K \phi(n)$. Write $\lambda_{n}=\{1+o(1)\} \phi(n)$, as $n \rightarrow \infty$. Then

$$
S_{3}>\frac{K}{x} \sum_{\pi / 2 x \leqq n \leqq 3 \pi / 2 x} \Delta \lambda_{n} \geqq \frac{K}{x}\left\{\lambda_{[\pi / 2 x]+1}-\lambda_{[3 \pi / 2 x]}\right\}>\frac{K}{x} \phi\left(\frac{1}{x}\right),
$$

as $x \rightarrow+0$. Hence $g(x)>(K / x) \phi(1 / x)$, as $x \rightarrow+0$. 
LEMMA 2. If $\phi(x) \sim\langle-1,0\rangle$, then, for small positive $x$,

$$
\left|\sum_{1}^{\infty} \phi(n) \cos n x\right| \leqq \frac{K}{x} \phi\left(\frac{1}{x}\right),
$$

and also, for any positive integer $N$,

$$
\left|\sum_{1}^{N} \phi(n) \cos n x\right| \leqq \frac{K}{x} \phi\left(\frac{1}{x}\right),
$$

where $K$ in (2.8) is independent of $N$.

The proofs of (2.7) and (2.8) are similar. For brevity, we only prove (2.7) here. Since $\phi(x) \sim\langle-1,0\rangle$, there exists $\varepsilon>0$, such that $x^{1-\varepsilon} \phi(x)$ increases and $x^{\varepsilon} \phi(x)$ decreases in $(0, \infty)$. By differentiating these functions we obtain

$$
\varepsilon \phi(x) / x \leqq-\phi^{\prime}(x) \leqq(1-\varepsilon) \phi(x) / x,
$$

where $\phi^{\prime}(x)$ exists almost everywhere. It follows as in the proof of Lemma 1 that

say. Here we have

$$
f(x)=\sum_{1}^{\infty} \phi(n) \cos n x=\sum_{1 \leqq n \leqq[1 / x]}+\sum_{n>[1 / x]}=S_{1}+S_{2},
$$

$$
\begin{aligned}
\left|S_{1}\right| & \leqq K \int_{1}^{1 / x} \phi(t) d t \leqq \frac{K}{x} \phi\left(\frac{1}{x}\right)-K \int_{1}^{1 / x} t \phi^{\prime}(t) d t \\
& \leqq \frac{K}{x} \phi\left(\frac{1}{x}\right)+K(1-\varepsilon) \int_{1}^{1 / x} \phi(t) d t \leqq \frac{K}{x} \phi\left(\frac{1}{x}\right)
\end{aligned}
$$

where the last inequality is obtained by shifting the term $K(1-\varepsilon) \int \ldots$ to combine with $K \int_{1}^{1 / x} \phi(t) d t$. Also, as in the proof of Lemma 1, we have $\left|S_{2}\right|<K x^{-1} \phi\left(x^{-1}\right)$. The result follows.

LEMMA 3. If $\lambda_{n} \geqq 0$, and if the series $\sum_{1}^{\infty} \lambda_{n} \sin n x$ converges everywhere to a function $g(x)$ such that $x^{-1} g(x) \in L(0, \pi)$, then $\sum_{1}^{\infty} \lambda_{n}<\infty$.

LEMMA 4. If $\lambda_{n} \geqq 0$ and if the series $\sum_{1}^{\infty} \lambda_{n} \sin n x$ converges everywhere to the function $f(x)$, such that $\phi(x) f(x) \in L(0, \pi)$, where $\phi(x) \sim\langle-1,0\rangle$, then

$$
\sum_{1}^{\infty} \frac{1}{n} \phi\left(\frac{1}{n}\right) \lambda_{n}<\infty .
$$

Lemma 3 is due to R. P. Boas [1]. For the proof of Lemma 4, it is sufficient to prove that the $n$th partial sum of $(2.9)$ is bounded. Write $\psi(x)=x^{-1} \phi\left(x^{-1}\right) \sim\langle-1,0\rangle$. The con- 
dition $\phi(x) f(x) \in L(0, \pi)$ with $\phi(x) \sim\langle-1,0\rangle$ implies that $\left.\phi(x) \geqq K_{\phi}\right\rangle 0$ in $(0, \pi)$ and that $f(x) \in L(0, \pi)$. Using Lemma 2 , we see that

$$
\begin{aligned}
& \sum_{1}^{n} \frac{1}{k} \phi\left(\frac{1}{k}\right) \lambda_{k}=\sum_{1}^{n} \psi(k) \lambda_{k}=\frac{2}{\pi} \sum_{1}^{n} \psi(k) \int_{0}^{\pi} f(x) \cos k x d x \\
& =\frac{2}{\pi} \int_{0}^{\pi} f(x) \sum_{1}^{n} \psi(k) \cos k x d x \leqq \frac{2}{\pi} \int_{0}^{\pi}|f(x)|\left|\sum_{1}^{n} \psi(k) \cos k x\right| d x \\
& \leqq K \int_{0}^{\pi} \frac{1}{x} \psi\left(\frac{1}{x}\right)|f(x)| d x=K \int_{0}^{\pi} \phi(x)|f(x)| d x<K .
\end{aligned}
$$

LEMMA 5. If $\phi(x) \sim\langle-2,-1\rangle$, and if

$$
g(x)=\sum_{1}^{\infty} \phi(n) \sin n x,
$$

then $g(x) \asymp x^{-1} \phi\left(x^{-1}\right)$.

Here it should be remarked that $x^{-1} \phi\left(x^{-1}\right)$ tends to zero as $x \rightarrow+0$. So it is not obvious that $\phi(n)$ in (2.10) can be replaced by $\lambda_{n} \simeq \phi(n)$, as in (2.1) of Lemma 1 .

Since $\phi(x) \sim\langle-2,-1\rangle$, we have $n \phi(n) \rightarrow 0$, as $n \rightarrow \infty$. By [7, Chap. 5, (1.3)], we see that $g(x) \rightarrow 0$, as $x \rightarrow+0$. On the other hand,

$$
g^{\prime}(x)=\sum_{1}^{\infty} n \phi(n) \cos n x=\sum_{1}^{\infty} \psi(n) \cos n x,
$$

where $\psi(x) \sim\langle-1,0\rangle$, and the series $(2.11)$ converges uniformly in $(\delta, \pi)$ for any $\delta>0$. It follows from Lemma 2 that

$$
g(x)=\lim _{\delta \rightarrow+0} \int_{\delta}^{x} g^{\prime}(t) d t=o\left\{\int_{0}^{x} \frac{1}{t} \psi\left(\frac{1}{t}\right) d t\right\}=o\left\{\int_{0}^{x} \chi(t) d t\right\}
$$

as $x \rightarrow+0$, where $\chi(t)=t^{-1} \psi\left(t^{-1}\right) \sim\langle-1,0\rangle$. Then, as in the proof of Lemma 2, we see that the right-hand member of $(2.12)$ is $O\{x \chi(x)\}=O\left\{x^{-1} \phi\left(x^{-1}\right)\right\}$.

Furthermore, it follows as in the proof of Lemma 1 that $g(x)>K x^{-1} \phi\left(x^{-1}\right)$. Thus the proof of Lemma 5 is completed.

3. We come now to the proof of Theorem 1. The argument is similar to the proof of Theorem 1 in [3]. For any positive integer $N$, write

$\frac{\pi}{2} \sum_{1}^{N-1} \phi(n) b_{n}=\int_{0}^{\delta} g(x) \sum_{1}^{N-1} \phi(n) \sin n x d x+\int_{\delta}^{\pi} g(x) \sum_{1}^{\infty} \phi(n) \sin n x d x$

$$
\begin{aligned}
& \quad-\int_{\delta}^{n} g(x) \sum_{N}^{\infty} \phi(n) \sin n x d x-\int_{\eta}^{\pi} g(x) \sum_{N}^{\infty} \phi(n) \sin n x d x \\
& =I_{1}+I_{2}+I_{3}+I_{4},
\end{aligned}
$$


say. Take $\delta=1 / N, \eta=1 / \sqrt{ } N$. We shall see that $I_{1}, I_{3}, I_{4}$ tend to zero and $I_{2}$ tends to a finite limit. In view of Lemmas 1 and 5, it follows from the hypothesis $x^{-1} g(x) \phi\left(x^{-1}\right) \in L(0, \pi)$ that the expression

$$
\lim _{N \rightarrow \infty} I_{2}=\lim _{\delta \rightarrow+0} \int_{\delta}^{\pi} g(x) \sum_{1}^{\infty} \phi(n) \sin n x d x
$$

is finite. Similarly, in view of (2.5) and Lemma 1,

$$
\left|I_{1}\right| \leqq \int_{0}^{\delta}|g(x)|\left|\sum_{1}^{N-1} \phi(n) \sin n x\right| d x \leqq K \int_{0}^{\delta} \frac{1}{x} \phi\left(\frac{1}{x}\right)|g(x)| d x=o(1),
$$

as $N \rightarrow \infty$. By similar arguments, it is easy to show that $I_{3}=o(1)$ and $I_{4}=o(1)$, as $N \rightarrow \infty$. This completes the proof of Theorem 1. Similar arguments apply in the proof of Theorem 2. The result follows in a similar way, except that Lemma 1 is replaced by Lemma 2 . Here we cannot replace $\phi(x) \sim\langle-1,0\rangle$ by $\phi(x) \sim[-1,0\rangle$. This may easily be seen from the special case $\phi(x)=1 / x$, where $\sum n^{-1} \cos n x \sim-\log x$, as $x \rightarrow+0$ [3, p. 174]. This also means that Theorem 2 does not hold for the case $\phi(x) \sim\langle-2,-1\rangle$.

For the proof of Theorem 3, we write

where

$$
\chi(x)=g(x)-\psi(x)
$$

$$
\psi(x)=\sum_{1}^{N} b_{n} \sin n x, \quad N=\left[\delta^{-1}\right]
$$

and

Let

$$
\int_{0}^{\pi} \phi(x) g(x) d x=\int_{0}^{\pi} \phi(x) \psi(x) d x+\int_{0}^{\pi} \phi(x) \chi(x) d x .
$$

$$
X(x)=\int_{0}^{x} \chi(t) d t .
$$

From Lemma 1 in [2], we see that $\phi(x)$ is absolutely continuous in $[\delta, \pi]$ for any $\delta>0$. Since $g(x) \in L(0, \pi)$ implies $\chi(x) \in L(0, \pi)$, integration by parts gives

$$
\int_{\delta}^{\pi} \phi(x) \chi(x) d x=\phi(\pi) X(\pi)-\phi(\delta) X(\delta)-\int_{\delta}^{\pi} \phi^{\prime}(x) X(x) d x .
$$

By similar arguments as in [3], it can readily be shown that the first and second terms on the right tend to zero as $\delta \rightarrow 0$. It remains to show that the last term tends to zero as $N \rightarrow \infty$. In fact, since $\phi(x) \sim\langle-2,0\rangle$ implies that $\phi(x)$ is absolutely continuous, it follows that

$$
\begin{aligned}
\left|\int_{0}^{\pi} \phi^{\prime}(x) X(x) d x\right| \leqq \int_{\delta}^{\pi}\left\{-\phi^{\prime}(x)\right\}|X(x)| d x \leqq \sum_{N+1}^{\infty} n^{-1}\left|b_{n}\right| \int_{\delta}^{\pi}\left\{-\phi^{\prime}(x)\right\} d x \\
=\sum_{N+1}^{\infty} n^{-1}\left|b_{n}\right|\{\phi(\delta)-\phi(\pi)\} \leqq \phi(\delta) \sum_{N+1}^{\infty} n^{-1}\left|b_{n}\right|=o(1),
\end{aligned}
$$


as $N \rightarrow \infty$, where $-\phi^{\prime}(x)$ is positive almost everywhere. Hence

$$
\int_{\delta}^{\pi} \phi(x) X(x) d x \rightarrow 0
$$

as $N \rightarrow \infty$. Then it is sufficient to consider

$$
\begin{aligned}
\int_{\delta}^{\pi} \phi(x) \psi(x) d x & =\int_{0}^{\pi} \phi(x) \psi(x) d x-\int_{0}^{\delta} \phi(x) \psi(x) d x \\
& =\int_{0}^{\pi} \phi(x) \psi(x) d x-\int_{0}^{\delta} \phi(x) \sum_{1}^{M} b_{n} \sin n x d x-\int_{0}^{\delta} \phi(x) \sum_{M+1}^{N} b_{n} \sin n x d x \\
& =J_{1}+J_{2}+J_{3},
\end{aligned}
$$

say, where $M=\left[\delta^{-\ddagger}\right]$. Write $\theta_{n}(t)=\phi(t / n) / \phi(1 / n)$ for $n=1,2,3, \ldots$ It is easy to see that

$$
t^{-\varepsilon} \leqq \theta_{n}(t) \leqq t^{e-2} \quad(0<t<1), \quad t^{\varepsilon-2} \leqq \theta_{n}(t) \leqq t^{-\varepsilon} \quad(t>1)
$$

Since $\theta_{n}(t)$ decreases steadily to zero as $t \rightarrow \infty$,

Hence

$$
\begin{aligned}
& \left|\int_{0}^{\pi} \phi(t) \sin n t d t\right|=\frac{1}{n} \phi\left(\frac{1}{n}\right)\left|\int_{0}^{n \pi} \theta_{n}(t) \sin t d t\right| \\
& \leqq \frac{1}{n} \phi\left(\frac{1}{n}\right)\left|\left(\int_{0}^{1}+\int_{1}^{\pi}\right) \theta_{n}(t) \sin t d t\right| \\
& \leqq \frac{1}{n} \phi\left(\frac{1}{n}\right)\left\{\int_{0}^{1} t^{e-2+1} d t+\int_{1}^{\pi} t^{-e} d t\right\} \leqq \frac{K}{n} \phi\left(\frac{1}{n}\right) .
\end{aligned}
$$

$$
J_{1}=\sum_{1}^{N} b_{n} \int_{0}^{\pi} \phi(x) \sin n x d x \rightarrow \sum_{1}^{\infty} b_{n} \int_{0}^{\pi} \phi(x) \sin n x d x,
$$

as $N \rightarrow \infty$, where the last series converges absolutely. It remains to estimate $J_{2}$ and $J_{3}$. We have

and

$$
\begin{aligned}
\left|J_{2}\right| & \leqq \sum_{1}^{[\sqrt{N}]}\left|b_{n}\right| \frac{1}{n} \phi\left(\frac{1}{n}\right) \int_{0}^{[1 / \sqrt{ } N]} \theta_{n}(t) \sin t d t \\
& \leqq K N^{-e / 2} \sum_{1}^{\infty} n^{-1} \phi\left(n^{-1}\right)\left|b_{n}\right|=o(1), \\
\left|J_{3}\right| & \leqq \sum_{[\sqrt{ } N]}^{N} n^{-1} \phi\left(n^{-1}\right)\left|b_{n}\right| \int_{0}^{n / N} \theta_{n}(t) \sin t d t \\
& \leqq \sum_{[\sqrt{ } N]}^{N} n^{-1} \phi\left(n^{-1}\right)\left|b_{n}\right| \int_{0}^{1} t^{e-1} d t \\
& \leqq K \sum_{[\sqrt{ } N]}^{\infty} n^{-1} \phi\left(n^{-1}\right)\left|b_{n}\right|=o(1),
\end{aligned}
$$

as $N \rightarrow \infty$. This completes the proof of Theorem 3 . 
Finally, it should be remarked that the proof of Theorem 4 is practically the same as that of Theorem 3. Using Lemma 3 and Lemma 4, the proof of Theorem 5 follows in a similar way as in [6] and is omitted here.

My thanks are due to the referee for pointing out a number of slips and for valuable suggestions.

\section{REFERENCES}

1. R. P. Boas, Jr, Integrability of trigonometric series (III). Quart. J. Math. Oxford Ser. (2) 3 (1952), 217-221.

2. Y. M. Chen, On two functional spaces, Studia Math. 24 (1964), 61-88.

3. P, Heywood, Integrability theorems for trigonometric series, Quart.J. Math. Oxford Ser. (2) 13 (1962), $172-180$. $38-53$.

4. J. Karamata, Sur un mode de croissance regulière des fonctions, Mathematica (Cluj) 4 (1930),

5. J. Karamata, Sur un mode de croissance regulière, Bull. Soc. Math. de France 61 (1933), 55-62.

6. Siobhan O'Shea, Note on an integrability theorem for sine series, Quart. J. Math. Oxford Ser. (2) 8 (1957), 279-281.

7. A. Zygmund, Trigonometric series, 2nd edn, Vol. 1 (Cambridge, 1959).

Department of Mathematics

UNIVERSITY OF HONG KONG 